\title{
Probiotics and their effects in reduction hyperlipidemia
}

\author{
Negin Farhad ${ }^{1}$, Somayeh Shahsavari ${ }^{2 *}$ \\ ${ }^{1}$ Department of Pathology, School of Medicine, Kermanshah University of Medical Sciences, Kermanshah, Iran \\ ${ }^{2}$ Biotechnology and Medicinal Plants Research Center, Ilam University of Medical Sciences, Ilam, Iran
}

\author{
*Correspondence to: \\ Somayeh Shahsavari \\ somayeh.shahsavari.pbp@ \\ gmail.com
}

Received: 3 June 2020

Accepted: 28 June 2020

ePublished:20 Novamber 2020

Keywords: Hyperlipidemia, Probiotics, Drug-therapy

\section{Citation:}

Farhad N, Shasavari S. Probiotics and their effects in reduction hyperlipidemia. Plant Biotechnol Persa 2020; 2(2): 21-23.

Copyright (c) 2020 The Author(s). This is an open-access article distributed under the terms of the Creative Commons Attribution License (http://creativecommons.org/licenses/by/4.0), which permits unrestricted use, distribution, and reproduction in any medium, provided the original work is properly cited. the antibiotics side effects; remission of arthritis rheumatoid patients; improving patients of helicobacter jejuni; reduction of cholesterol and hypertension; preventing cancer of colon, liver, bowl, breast; and enhancing lactose intolerance $[12,13]$. The purpose of this review is to assess clinical evidences associating to role and effects of probiotics on the hl.

\section{Method for review}

In this review, the intended articles were evaluated by searching the keywords including probiotic and blood lipid, probiotic, and clinical trial in the time period of 1999 to 2014.

\section{Results}

Among studies and clinical trials articles we got eleven articles linked to the effects of probiotics on hl. The results of clinical trials related to the influences on blood lipid are listed in table 1. ics are alive microorganisms which have useful effects on the health and can control pathogenic microbes. The most common probiotics are lactic acid bacteria including bifidobacter species and lactobacillus $[10,11]$. Consuming the probiotics may enhance hl and shorten the disease course and reduce its signs. Moreover, these compounds cause improvement in maximum oxygen intake in athletes. Among the other results of probiotics, are as follows: decreasing 
Lactobacillus gasseri

Lactobacillus plantarum

Probiotic

Lactobacillus paracasei

Lactobacillus gasseri

Bifidobacterium longum

Enterococcus faecium

Lactobacillus acidophilus and Bifidobacterium lactis

Lactobacillus acidophilus and Consumption of yogurt containing 3x108 lactobacillus acidophilus and bifidobacterium lactis for Bifidobacterium 6 weeks reduced total cholesterol significantly. lactis

Probiotic

Intake of probiotic yogurt for 9 weeks resulted in significant reduction in total cholesterol, LDL-C, HDL-C and triglycerides in pregnant women.

Probiotic

Prescription of fermented milk containing 1011 lactobacillus gasseri for 12 weeks results in significant decrease in abdomen, visceral and subcutaneous fat in obese people at the end of intervention course.

Taking 50 g probiotic cheese containing 108 bacteria lactobacillus plantarum per gram reduced $\mathrm{BMI}$ in obese people with hypertension, significantly.

Prescription of probiotic yogurt-diverse probiotic species- in different doses for 8 weeks decreased body mass index in obese persons with metabolic syndrome, significantly.

Prescribing sausages containing 5×109 lactobacillus paracasei for 5 weeks, Jahreis et al. did not observe significant changes in lipid profiles of people suffering from hypercholesterolemia; but, oxidized anti-LDL-C antibodies increased significantly at the end of intervention period.

Administration of supplementary containing 1010 lactobacillus gasseri for 12 weeks for high weight and obese adults resulted to significant reduction in weight, waist size and hip size compared with control group.

Daily intake of 9x1010 bifidobacterium longum for 4 weeks could result in marked decrease in total cholesterol especially in people with mild hypercholesterolemia.

Receiving enterococcus faecium by healthy people for 56 weeks caused reduction in total cholesterol by $12 \%$ and decrease in LDL cholesterol by $19 \%$; but no significant changes were observed in HDL-C and triglycerides.

Daily prescription of $300 \mathrm{~g}$ probiotic yogurt containing 100 lactobacillus acidophilus and 109 bifidobacterium lactis for 6 weeks resulted in significant drop in total cholesterol, LDL-C, total cholesterol toward HDL-C relation, and LDL-C to HDL-C in diabetic people.

Prescription of probiotic yogurt-diverse probiotic species- in different doses for 8 weeks caused significant decrease in total cholesterol and LDL-C in people suffering from metabolic syndrome.

\section{Discussion}

The mechanisms including reducing intake from bowl and de-conjugation of bile acids leading to their reduced ingestion of them in bowl, entry of a fraction of received fats from foods into these microorganisms' cell membrane, and converting some of cholesterol to indigestible coprostanol are routines of mechanisms to reduction effects of probiotic cholesterol $[17,18]$. On the other hand, short chain fatty acids produced from probiotics may inhibit hydroxyl methyl glutarate coenzyme A reductase an important enzyme in the pathway for synthesis of cholesterol in liver- and finally decrease the production of cholesterol in liver [19-23]. Fatty acid metabolism may be undergo alterations by the bowl microflora; lactobacillus and Bifidobacteria may convert the free linoleic acid to conjugated type. The conjugated linoleic acid has properties including antidiabetic, anti-sclerosis, enhancing immune system and anti-obesity [16]. Bacteria residing in bowl ferments indigestible carbohydrates into short chain fatty acids (acetate, propionate and butyrate). These compounds bind to G-protein-coupled receptors and cause secretion of PYY intestinal hormone. This hormone reduces the intestinal movements so, enhances the foods absorption from the intestine [19-22]. Probiotics have higher influence on people with hyper- cholesterolemia. In bowl, probiotics may bind to cholesterol and inhibit its ingestion. Furthermore, they help to production of some bile acids that have role in fat and cholesterol metabolism in body. Some probiotics are able to produce short chain fatty acids which may help to inhibit the production of cholesterol in liver. Finally, they are important factors in reduction of blood lipids.

\section{Authors' contribution}

All authors contributed equally to the manuscript.

\section{Conflicts of interest}

The authors declared no competing interests.

\section{Ethical considerations}

Ethical issues (including plagiarism, data fabrica $\neg$ tion, double publication and etc.) have been completely ob-served by author.

\section{Funding/Support}

None. 


\section{Referenceses}

1. World Health Organization (WHO). Cardiovascular disease (CVDs), 2009. www.who.int/mediacentre/factsheets/fs317/ en/index.html (accessed 30 December 2010).

2. World Health Organization Statistical Information System (WHOSIS) 2009. Statistical Information System. www.who. int/whosis/en/ (accessed 30 December 2010).

3. Athyros VG, Tziomalos K, Karagiannis A, Mikhailidis DP. Dyslipidaemia of obesity, metabolic syndrome and type 2 diabetes mellitus: the case for residual risk reduction after statin treatment. The Open Cardiovascular Med J. 2011; 5:24-34.

4. Go AS, Mozaffarian D, Roger VL, et al. Heart disease and stroke statistics-2013 update: a Report from the American Heart Association. Circulation. 2013; 127(1): 6-245.

5. Kusters DM, Avis HJ, Braamskamp MJ, et al. Inheritance pattern of familial hypercholesterolemia and markers of cardiovascular risk. J Lipid Res. 2013; 54(9): 2543-2549.

6. Talmud PJ, Shah S, Whittall R, et al. Use of low-density lipoprotein cholesterol gene score to distinguish patients with polygenic and monogenic familial hypercholesterolaemia: a case-control study. Lancet. 2013; 381(9874):1293-1301.

7. Goldstein JL, Schrott HG, Hazzard WR, Bierman EL, Motulsky AG. Hyperlipidemia in coronary heart disease. II. Genetic analysis of lipid levels in 176 families and delineation of a new inherited disorder, combined hyperlipidemia. J Clin Invest. 1973; 52(7): 1544-68.

8. Fredrickson DS, Levy RI, Lees RS. Fat transport in lipoproteins-an integrated approach to mechanisms and disorders. New England J Med. 1967; 276(1):34-42.

9. Toth PP. Drug treatment of hyperlipidaemia: a guide to the rational use of lipid-lowering drugs. Drugs. 2010; 70(11):1363-1379.

10. Mortazavian AM, Razavi SH, Ehsani M, Sohrabvandi S. Principles and methods of microencapsulation of probiotic microorganisms. Iran J Biotechnol. 2007; 5 (1): 1-18.

11. Vinderola CG, Reinheimer JA. Enumeration of Lactobacilllus casei in the presence of I. acidophilus, bifidobacteria and lactic starter bacteria in fermented dairy products. Int Dairy J. 2000; 10: 271-275.

12. Mortazavian AM, Ehsani MR, Mousavi SM, Reinheimer JA, Sohrabvandi S, et al. Preliminary investigation of the combined effect of heat treatment and incubation temperature on the viability of the probiotic micro- organisms in freshly made yogurt. Int J Dairy Tech. 2006; 59 (1).

13. Mortazavian A, Sohrabvandi S. A textbook of review of probiotic and probiotic products. 1th ed. Tehran: Ata publisher; 2006; 48.

14. Kadooka Y, Sato M, Imaizumi K, Ogawa A, Ikuyama K, Akai $\mathrm{Y}$, et al. Regulation of abdominal adiposity by probiotics (Lactobacillus gasseri SBT2055) in adults with obese tendencies in a randomized controlled trial. Euro J Clin Nutr. 2010; 64(6):636-43.

15. Sharafedtinov KK, Plotnikova OA, Alexeeva RI, Sentsova TB, Songisepp E, Stsepetova J, et al. Hypocaloric diet supplemented with probiotic cheese improves body mass index and blood pressure indices of obese hypertensive patients- $A$ randomized double-blind placebo-controlled pilot study. Nutr J. 2013; 12(1):138-48.

16. Chang B, Park $S$, Jang $Y$, Ko $S$, Joo N, Kim S, et al. Effect of functional yogurt NY-YP901 in improving the trait of metabolic syndrome. Euro J Clin Nutr. 2011; 65(11):1250-5.

17. Jahreis 0, Vogelsang H, Kiessling G, Schubert R, Bunte C, Hammes W. Influence of probiotic sausage (Lactobacillus paracasei) on blood lipids and immunological parameters of healthy volunteers. Food Res Intern. 2002; 35(2):133-8.

18. Jung S-P, Lee K-M, Kang J-H, Yun S-I, Park H-0, Moon Y, et al. Effect of Lactobacillus gasseri BNR17 on overweight and obese adults: a randomized, double-blind clinical trial. Korean J Family Med. 2013; 34(2):80-9.

19. Xiao J, Kondo S, Takahashi N, Miyaji K, Oshida K, Hiramatsu A, et al. Effects of milk products fermented by Bifidobacterium longum on blood lipids in rats and healthy adult male volunteers. J Dairy Sci. 2003; 86(7): 2452-61.

20. Hlivak P, Odraska J, Ferencik M, Ebringer L, Jahnova E, Mikes Z. One-year application of probiotic strain Enterococcus faecium M-74 decreases serum cholesterol levels. Bratisl Lek Listy. 2005; 106(2):67-72.

21. Ejtahed H, Mohtadi-Nia J, Homayouni-Rad A, Niafar $M$, Asghari-Jafarabadi M, Mofid V, et al. Effect of probiotic yogurt containing Lactobacillus acidophilus and Bifidobacterium lactis on lipid profile in individuals with type 2 diabetes mellitus. J Dairy Sci. 2011; 94(7): 3288-94.

22. Ataie-Jafari A, Larijani B, Alavi Majd H, Tahbaz F. Cholesterol-lowering effect of probiotic yogurt in comparison with ordinary yogurt in mildly to moderately hypercholesterolemic subjects. Annals of Nutr Metabol. 2009; 54(1):22-7.

23. 23. Asemi Z, Samini M, Tabasi Z, talebian P, Azarbaz Z, Heydarzadeh $Z$ et al. Effect of daily consumption of probiotic yoghurt on lipid profile in pregnant women: a randomized control clinical trial. The J Maternal-Fetal Neonatal Med. 2012; 25(9): 1552-9. 